\title{
Alongamento agudo da musculatura agonista $x$ antagonista no desempenho funcional do membro inferior
}

\author{
Acute stretching of the agonist $x$ antagonist muscles in lower limb functional performance
}

\author{
Victória Marianne Araújo Valadão ${ }^{1, *}$ \\ Caio de Paula Martins ${ }^{2}$ \\ Rafael Mian da Silva ${ }^{2}$ \\ Daniel Ferreira Moreira Lobato ${ }^{1}$
}

\section{Resumo}

Objetivo:Comparar o efeito do alongamento muscular agudo da musculatura agonista e antagonista em membros inferiores sobre a performance em testes de salto. Método: 0 estudo envolveu 20 atletas futebolistas, do sexo masculino, com idade entre 14 e 17 anos, divididos aleatoriamente em dois grupos: 1) submetidos ao alongamento agudo da musculatura agonista (extensores de quadril e joelho e flexores plantares de tornozelo $-n=10$ ) e 2) submetidos ao alongamento agudo da musculatura antagonista (flexores de quadril e joelho e dorsiflexores de tornozelo $-n=10$ ). Um dos membros inferiores foi sorteado para receber a intervenção e o membro contralateral foi considerado como controle interno. O desempenho funcional foi avaliado por meio do triple hop test e six-meter timed hop test, antes e após a realização dos alongamentos ( 3 séries de 30 segundos), considerando-se o melhor resultado obtido em 3 tentativas válidas de cada teste. Resultados: Não houve diferença significativa $(\alpha=5 \%)$ entre o desempenho pré e pós-alongamento, tanto da musculatura agonista quanto da musculatura antagonista, em ambos os testes, assim como para o membro inferior controle. Conclusões: A realização do alongamento agudo na musculatura agonista e antagonista parece não interferir na performance dos atletas em atividades de salto.

Palavras-chave: exercícios de alongamento muscular, modalidades de fisioterapia, desempenho atlético.

\begin{abstract}
Objective: To compare the effects of acute agonist and antagonist lower limb muscle stretching on hop tests functional performance. Methods: Twenty soccer male athletes, aged between 14 and 17 years, were divided into two groups: 1) who were submitted to acuteagonist (hip and knee extensors and ankle plantar flexors $-n=10$ ) muscles stretching and 2) who were submitted to acute antagonist (hip and knee flexors and ankle dorsiflexors $-n=10$ ) muscles stretching. One of the lower limbs was drawn to receive the intervention and the contralateral limb was considered as internal control. Functional performance was evaluated by the triple hop test and six-meter timed hop test, before and after performing thes tretches ( 3 sets of 30 seconds), considering the best result obtained in 3 valid attempts of each test. Results: There was no significant difference $(\alpha=5 \%)$ between the pre and post stretching performance of both the agonist and the antagonist muscles in both tests, as well as for the control limb. Conclusion: The acute agonist and antagonist muscles stretching does not seem to modify the athletes' jump performance.
\end{abstract}

Keywords: muscle stretching exercises, physical therapy modalities, athletic performance.
Afiliação dos autores

${ }^{1}$ Universidade Federal do Triângulo Mineiro, Uberaba, Minas Gerais, Brasil.

${ }^{2}$ Centro Universitário UNISEB, Ribeirão Preto, São Paulo, Brasil.

${ }^{\star}$ Autor correspondente

Rua Vigário Carlos, 100, Bloco B, 4 andar, Sala 406, Abadia, CEP 38025-350, Uberaba, Minas Gerais, Brasil.

e-mail: vivimarianne4@gmail.com

Conflito de interesses

Os autores declararam não haver conflito de interesses.

Processo de arbitragem

Recebido: 27/12/2018 Aprovado: 03/03/2019 


\section{Introdução}

O alongamento muscular é frequentemente utilizado nas práticas desportivas, com o objetivo de aumentar a flexibilidade muscular e a amplitude de movimento articular, assim como tentativa de diminuir o risco de lesões e, possivelmente, melhorar o desempenho atlético ${ }^{1}$. Existe uma variedade de técnicas de alongamento, dentre as quais destaca-se 0 alongamento estático, o alongamento dinâmico e o alongamento por facilitação neuromuscular proprioceptiva - FNP ${ }^{2}$.

Apesar do método por FNP ser proposto como mais eficaz, o alongamento estático é o mais utilizado, pois é o método mais seguro, simples e com menor risco de lesão. No método estático, o membro é mantido em posição estacionária no maior comprimento muscular possível por um período de 15 a 60 segundos ${ }^{4}$. Este procedimento deve ser realizado de forma lenta e gradual, para evitar a resposta neural do fuso neuromuscular, via reflexo do estiramento, bem como estimular a atividade dos órgãos tendinosos de Golgi, inibindo a contração do músculo alongado ${ }^{5}$

Percebe-se que a prática do alongamento estático agudo, ou seja, aquele realizado imediatamente antes dos exercícios, vem sendo alvo de controvérsias no âmbito científico em relação aos seus benefícios, no que diz respeito ao desempenho muscular e prevenção de lesões ${ }^{2}$. Diversos estudos ${ }^{6-9}$ foram realizados a fim de averiguar os efeitos do alongamento muscular antes do exercício. Contudo, nota-se a falta de consenso entre os seus resultados ${ }^{2}$.

Ao desenvolver uma revisão crítica sobre o tema, Shrier ${ }^{10}$ constatou que, em 20 estudos, o alongamento não foi benéfico para o desempenho em eventos relacionados à força, à produção de torque $e$ aos saltos. Tais achados são concordantes a outros ${ }^{6,9,11}$ que apontam que $\mathrm{o}$ alongamento agudo e prolongado, executado imediatamente antes do exercício, pode reduzir a capacidade de produzir força e a potência muscular. Especula-se que fatores neurais $e$ mecânicos estariam envolvidos na redução temporária da atividade e da força muscular ${ }^{12}$ tais como a redução no recrutamento de unidades motoras, ativação dos órgãos tendinosos de Golgi e contribuição dos nociceptores, além de modificações na relação comprimento-tensão dos sarcômeros, com alteração da sobreposição fisiológica entre os filamentos de actina e miosina ${ }^{13}$.

Por outro lado, alguns estudos ${ }^{14,15}$ demonstraram que o treinamento de flexibilidade pode melhorar a performancenos exercícios de força envolvendo o ciclo alongamentoencurtamento muscular, ou mesmo não exercer influência significativa no rendimento físico ${ }^{16,17}$.

Entretanto, a maioria dos estudos consultados na literatura dedicaram-se a verificar os efeitos do alongamento agudo da musculatura agonista à ação a ser desempenhada, e poucos estudos $^{1,18,19}$ investigaram os efeitos do alongamento agudo da musculatura antagonista sobre a força e a potência muscular. Acredita-se que o alongamento da musculatura antagonista possa resultar em sua inibição e, reciprocamente, facilitar o aumento da atividade da musculatura agonista, com subsequentes melhorias na força e potência muscular.

Neste sentido, esses estudos evidenciaram que 0 alongamento agudo da musculatura antagonista resultou em uma melhora significativa no desempenho atlético, tanto nos testes de salto ${ }^{1,19}$, no torque extensor do joelho ${ }^{1}$ e no desempenho muscular dos agonistas ${ }^{1,18}$. Entretanto, os autores concluem que ainda não há justificativas conclusivas que comprovem a efetividade do alongamento da musculatura antagonista para melhorar o desempenho em atividade de alta velocidade.

Diante do exposto, verifica-se que, embora o tema tenha sido investigado por diversos estudos, o mesmo ainda propõe discussões, em decorrência da controvérsia de resultados encontrados quanto às formas de alongamento tradicional (musculatura agonista) e recentemente sugerida (musculatura antagonista).Portanto este estudo teve como objetivo verificar o efeito do alongamento muscular agudo da musculatura agonista e da musculatura antagonista, de forma comparativa, sobre o desempenho funcional de atletas profissionais.

\section{Métodos}

Participantes
Participaram do estudo, de caráter transversal, vinte atletas amadores $(67,30 \pm 6,50 \mathrm{~kg} ; 1,73 \pm 0,05 \mathrm{~m})$ de uma agremiação esportiva local, do sexo masculino, com idade entre 14 e 17 anos $(15,30 \pm 0,94$ anos) e submetidos a treinamentos diários, no período vespertino. Os atletas foram divididos aleatoriamente (por sorteio simples) em dois grupos: 1) submetidos ao alongamento agudo da musculatura agonista - GAG (extensores de quadril e joelho e flexores plantares de tornozelo) e 2) submetidos ao alongamento agudo da musculatura antagonista - GAN (flexores de quadril e joelho e dorsiflexores de tornozelo). A descrição do perfil demográfico e antropométrico dos voluntários, bem como as características relacionadas ao esporte e à dominância de membros, encontra-se detalhada na Tabela 1.

\section{Tabela 1}

Dados antropométricos e demográficos dos participantes deste estudo $(n=10)$.

\begin{tabular}{llll}
\hline & \multicolumn{1}{c}{ GAG } & \multicolumn{1}{c}{ GAN } & \multicolumn{1}{c}{$\begin{array}{c}\text { v- } \\
\text { valor }\end{array}$} \\
\hline Idade (anos) & $15,70 \pm 1,06$ & $15,30 \pm 0,95$ & 0,38 \\
Massa corporal (kg) & $62,40 \pm 7,06$ & $67,30 \pm 6,55$ & 0,12 \\
Estatura (m) & $1,71 \pm 0,07$ & $1,73 \pm 0,05$ & 0,36 \\
& 1 zagueiro, 5 & 1 goleiro, 3 & \\
Posição & laterais, 1 meia e 3 & zagueiros, 4 & \\
& atacantes & atantes e 2 & \\
Dominância & $60 \%$ (D) e 40\% (E) & $70 \%$ (D) e 30\% (E) & \\
Membro controle & $60 \%$ (D) e 40\% (E) & $70 \%$ (D) e 30\% (E) & \\
Membro alongado & $40 \%$ (D) e 60\% (E) & $30 \%$ (D) e 70\% (E) &
\end{tabular}

(D) agonista; GAN = grupo submetido ao alongamento agudo da musculatura antagonista; $\mathrm{D}=$ membro inferior direito; $\mathrm{E}=$ membro inferior esquerdo.

Foram considerados como critérios de exclusão: a) idade inferior a 14 anos e superior a 17 anos; b) condição de lesão em qualquer segmento corporal; c) presença de dores ou disfunções relacionadas aos complexos articulares do membro inferior; d) existência de cirurgias prévias no membro inferior e e) presença de disfunções cárdio-respiratórias que limitassem a realização da atividade. Todos os responsáveis pelos voluntários assinaram um Termo de Consentimento Livre e Esclarecido previamente à participação no estudo, sendo a pesquisa aprovada pelo Comitê de Ética institucional (parecer $\mathrm{n}^{\circ}$ 2.073.109/2017), e conduzida no ano de 2017.

\section{Procedimentos}

Os atletas foram submetidos a uma avaliação inicial para a coleta de dados clínicos e físicos, bem como para a verificação dos critérios de inclusão e exclusão no estudo. A dominância de membros foi determinada perguntando aos atletas qual o membro que eles utilizavam para chutar uma bola na máxima distância possível ${ }^{20}$. Por fim, foi sorteado qual membro (dominante ou não-dominante) seria submetido ao alongamento, sendo que o membro não alongado foi utilizado como controle.

O desempenho funcional foi avaliado por meio de dois testes de ampla utilização clínica: triple hop test e six-meter timed hop test. A ordem de realização dos testes também foi sorteada para cada voluntário. Os atletas receberam uma breve explicação sobre os testes funcionais e assistiram a uma demonstração de realização dos mesmos. Em seguida, foram submetidos a uma familiarização com cada teste, para garantir a adequada compreensão das ações requeridas e dos procedimentos envolvidos. Além disso, esse procedimento consistiu em uma medida de aquecimento específico para cada tarefa a ser realizada.

Para a realização do triple hop test (Figura $1 \mathrm{~A}$ ), os atletas foram instruídos a permanecer em apoio simples, imediatamente atrás de uma marcação inicial. Com os membros superiores livres ao lado do corpo, os atletas deveriam realizar três saltos consecutivos e máximos para atingir a máxima distância horizontal possível, aterrissando unicamente sobre o membro inferior que realizou o salto, com a medida de distância saltada (em metros) sendo registrada pelos examinadores por meio de uma fita métrica comum. O teste foi executado em três tentativas válidas, sendo os melhores resultados obtidos utilizados para a análise dos dados.

Os critérios para considerar uma tentativa válida foram a manutenção do equilíbrio em apoio unipodal, sobre o membro inferior que realizou o salto, por um período mínimo de 2 
segundos sem a perda de equilíbrio, sem tocar com a extremidade inferior contralateral ou com a extremidade superior no solo, ou ainda sem realizar um salto adicional durante a aterrissagem. Foi respeitado um intervalo de repouso de 30 segundos entre cada tentativa. O mesmo protocolo foi repetido para o membro contralateral.

Para a realização do six-meter timed hop test (Figura 1B), os atletas foram instruídos a permanecer em apoio simples imediatamente atrás de uma marcação inicial. Com os membros superiores livres ao lado do corpo, os atletas deveriam percorrer uma distância de seis metros (demarcada no local do exame por cones) saltando na máxima velocidade possível e sem desacelerar ao longo do teste, com a medida de tempo decorrido (em segundos) sendo registrada pelos examinadores por meio de um cronômetro digital. O teste foi executado em três tentativas válidas, sendo os melhores valores obtidos utilizados para a análise dos dados.

Os critérios para considerar uma tentativa válida foram a manutenção do equilíbrio ao longo da atividade, sobre o membro inferior que realizou os saltos, sem tocar com a extremidade inferior contralateral ou com a extremidade superior no solo, bem como a manutenção de um estado de aceleração máxima ao longo do teste. Foi respeitado um intervalo de repouso de 60 segundos entre cada tentativa. $\mathrm{O}$ mesmo protocolo foi repetido para o membro contralateral.

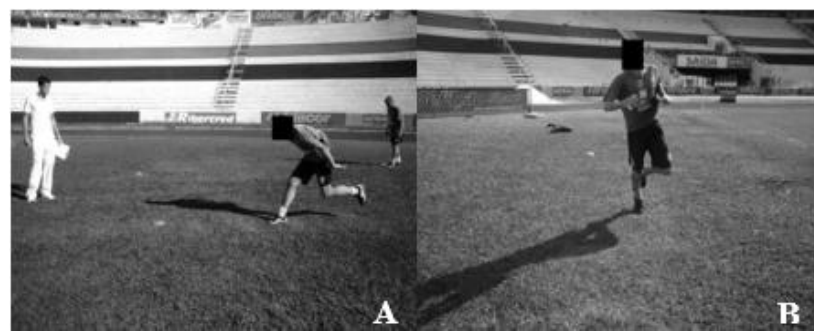

Figura 1. Realização dos testes: A) Triple hop test; B) Sex-meter timed hop test.

\section{Intervenção}

Os voluntários do GAG foram submetidos a um programa de alongamentos para a musculatura agonista às atividades funcionais avaliadas, ou seja, destinado aos músculos extensores do quadril, extensores do joelho e flexores plantares de tornozelo (Figura 2), enquanto os voluntários do GAN foram submetidos a um programa de alongamentos para a musculatura antagonista, destinado aos músculos flexores do quadril, flexores do joelho e dorsiflexores do tornozelo (Figura 3), conforme proposto por Sandberg et al. ${ }^{1}$.

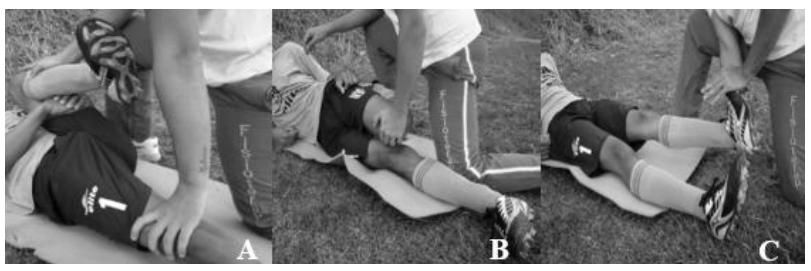

Figura 2. Demonstração dos alongamentos para a musculatura agonista: A) Extensores de quadril; B) Extensores de joelho e C) Flexores plantares do tornozelo.

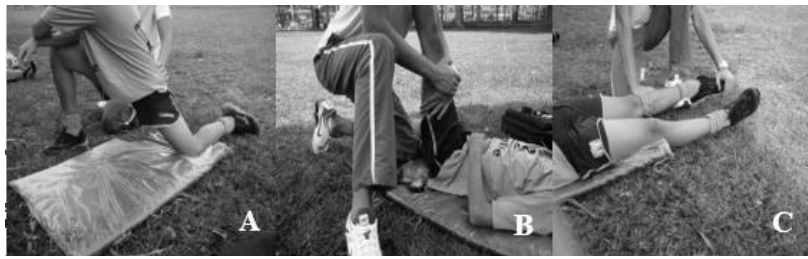

Figura 3. Demonstração dos alongamentos para a musculatura antagonista A) Flexores de quadril; B) Flexores de joelho e C) Dorsiflexores de tornozelo.

Cada exercício de alongamento foi realizado passivamente pelo examinador antes dos testes, por meio de 3 séries de 30 segundos de duração, com descanso de 20 segundos para cada grupo muscular. Após cada tentativa válida, foi realizada uma série de 30 segundos de alongamento, para os três grupos musculares, a fim de reforçar o caráter agudo dos alongamentos.

\section{Análise dos dados}

Os dados de desempenho funcional foram analisados por meio do software STATISTICA 7.0 for Windows (StatSoft, Inc, Tulsa, USA). Inicialmente foi verificada a normalidade dos conjuntos de dados pelo teste de Shapiro Wilks. De acordo com os resultados obtidos, foi utilizado o teste t-Student para amostras dependentes (dados paramétricos) para a comparação do desempenho funcional dos membros inferiores antes e após a intervenção realizada, considerando um nível de significância de $5 \%$.

\section{Resultados}

Os dados referentes ao desempenho funcional dos voluntários submetidos ao alongamento agudo da musculatura agonista encontram-se apresentados na Tabela 2. Não houve diferença significativa entre 0 desempenho pré e pósalongamento do membro submetido à intervenção, tanto no triple hop test $(p=0,66)$, quanto no six-meter timed hop test $(p=0,82)$. Da mesma forma, o membro controle não apresentou desempenho diferenciado entre a $1^{\underline{a}}$ e $2^{\underline{a}}$ avaliações, tanto no triple hop test $(p=0,24)$, quanto no six-meter timed hop test $(p=0,39)$. As variações percentuais entre a primeira e segunda avaliações corroboraram os achados estatísticos, indicando que em nenhuma das situações houve alteração clinicamente significativa (>10\%) do desempenho funcional.

\section{Tabela 2}

Valores obtidos THT e no SHT no grupo submetido à intervenção pelo alongamento agudo da musculatura agonista, bem como variação percentual entre as duas avaliações $(n=10)$

\begin{tabular}{ccccccc}
\hline \multirow{2}{*}{ Teste } & \multicolumn{3}{c}{ Membro alongamento } & \multicolumn{3}{c}{ Membro controle } \\
\cline { 2 - 7 } & Pré & Pós & Var $(\%)$ & Pré & Pós & Var (\%) \\
\hline \multirow{2}{*}{ THT $(\mathrm{m})$} & $6,00 \pm$ & $6,02 \pm$ & \multirow{2}{*}{$+0,3$} & $6,00 \pm$ & $5,95 \pm$ & $-0,8$ \\
& 0,49 & 0,61 & & 0,47 & 0,49 & \\
\multirow{2}{*}{ SHT $(\mathrm{s})$} & $1,79 \pm$ & $1,76 \pm$ & $-1,7$ & $1,87 \pm$ & $1,82 \pm$ & $-2,7$ \\
& 0,21 & 0,18 & & 0,27 & 0,20 & \\
\hline
\end{tabular}

Os dados referentes ao desempenho funcional dos voluntários submetidos ao alongamento agudo da musculatura antagonista encontram-se apresentados na Tabela 3. Não houve diferença significativa entre o desempenho pré e pósalongamento do membro submetido à intervenção, tanto no triple hop test $(\mathrm{p}=0,90)$, quanto no six-meter timed hop test $(p=0,63)$. Da mesma forma, o membro controle não apresentou desempenho diferenciado entre a $1^{\underline{a}}$ e $2^{\underline{a}}$ avaliações, tanto no triple hop test $(p=0,72)$, quanto no six-meter timed hop test $(p=0,63)$. As variações percentuais entre a primeira e segunda avaliações corroboraram os achados estatísticos, indicando que em nenhuma das situações houve alteração clinicamente significativa (>10\%) do desempenho funcional.

\section{Tabela 3}

Valores obtidos no THT e no SHT pelos membros submetidos à intervenção pelo alongamento agudo da musculatura antagonista e pelos membros controle, bem como variação percentual entre as duas avaliações $(n=10)$.

\begin{tabular}{ccccccc}
\hline \multirow{2}{*}{ Teste } & \multicolumn{3}{c}{ Membro alongamento } & \multicolumn{3}{c}{ Membro controle } \\
\cline { 2 - 7 } & Pré & Pós & $\begin{array}{l}\text { Var } \\
(\%)\end{array}$ & Pré & Pós & $\begin{array}{c}\text { Var } \\
(\%)\end{array}$ \\
\hline $\begin{array}{c}\text { THT } \\
\text { (m) }\end{array}$ & $, 01 \pm 0,54$ & $, 98 \pm 0,60$ & 0,5 & $, 82 \pm 0,42$ & $, 97 \pm 0,58$ & 2,6 \\
$\begin{array}{c}\text { SHT } \\
(\mathrm{s})\end{array}$ & $, 84 \pm 0,28$ & $, 81 \pm 0,23$ & 1,6 & $, 86 \pm 0,25$ & $, 94 \pm 0,23$ & 4,3 \\
\hline
\end{tabular}

\section{Discussão}

O presente estudo verificou o efeito do alongamento muscular agudo da musculatura agonista e da musculatura antagonista, de forma comparativa, sobre o desempenho funcional de atletas profissionais. Os resultados obtidos apontam que a realização do alongamento agudo tanto da musculatura agonista quanto antagonista não apresentou diferenças 
significativas entre o desempenho pré e pós alongamento do membro submetido à intervenção, assim como ocorrido no membro controle.

O alongamento muscular na prática desportiva tem sido muito discutido quanto a sua eficácia no momento pré e/ou pósexercício. Diversos estudos ${ }^{1,6,11,21-23}$ buscaram, de forma aplicada, comprovar os seus benefícios ou malefícios, ou mesmo verificar se o desempenho não é alterado em função da realização do alongamento agudo.

Recentemente foi publicada uma meta-análise sobre o assunto, com o objetivo de buscar evidências sobre a relação entre 0 alongamento e o desempenho funcional de atletas ${ }^{11^{3}}$ no qual foram revisados 104 artigos a respeito do alongamento muscular pré-exercício e os seus efeitos sobre a performance. Apesar do número crescente de estudos demonstrando uma diminuição na queda de desempenho ${ }^{24,25}$, há relatos de que não há alteração no desempenho ${ }^{26-28}$, e uma minoria de estudos indica uma melhora no rendimento ${ }^{7}$. Deste modo, os autores reportam que, de forma geral, o alongamento agudo préexercício parece levar a resultados negativos no desempenho, principalmente em testes isométricos, quando comparados com testes dinâmicos.

Poucos artigos foram encontrados na literatura comprovando que o alongamento agudo estático melhora a performance. Desses, destaca-se o estudo de Wordell et al. ${ }^{21}$, que buscou avaliar a relação entre flexibilidade e desempenho do grupo muscular dos isquiotibiais, utilizando a técnica de facilitação neuromuscular-proprioceptiva e alongamento estático em dezenove indivíduos. Este estudo demonstrou que houve um aumento significativo dos torques concêntrico e excêntrico após a realização do alongamento. Com resultados similares, O'Connor et al. ${ }^{7}$ avaliaram 27 indivíduos saudáveis, submetidos a duas sessões de testes (aquecimento e aquecimento + alongamento), concluindo que a segunda sessão (aquecimento + alongamento) resultou em um pico de potência mais rápido em relação à primeira sessão.

Shrier $^{10}$, em uma revisão sistemática de 23 artigos avaliando os efeitos de uma sessão aguda de alongamento, constatou que em 20 estudos houve efeitos negativos no desempenho para força isométrica, potência, altura e velocidade do salto e, em apenas um estudo ${ }^{29}$, houve resultados contrários. Com achados de mesma tendência, Dacardia et al. $^{6}$ verificaram que 0 alongamento agudo estático da musculatura agonista de membros inferiores levou a um decréscimo de rendimento funcional (salto vertical, salto horizontal e teste de velocidade de deslocamento) em atletas com idade média de 15 anos, portanto com perfil muito similar ao utilizado no presente estudo.

Uma das explicações mais aceitas para a redução na performance refere-se aos princípios fisiológicos do alongamento na atividade esportiva. A força de uma contração muscular depende do comprimento dos sarcômeros, segundo a teoria de relação comprimento-tensão das fibras musculares ${ }^{30}$. Quando os sarcômeros são distanciados até um comprimento maior, a zona de sobreposição entre os filamentos de actina e miosina diminui e, conseqüentemente, a tensão que pode ser gerada pela fibra também sofre redução. Quando a fibra muscular esquelética é estirada a $170 \%$ de seu comprimento ótimo, não existe mais sobreposição entre os filamentos finos (miosina) e grossos (actina), e então surge o denominado estiramento muscular. Ambas as situações são consideradas como desfavoráveis ao desempenho muscular, uma vez que estão associadas a um menor número de pontes cruzadas entre actina e miosina atuantes gerando, portanto, menor capacidade de produção de torque.

Aumentando a controvérsia de resultados sobre o tema, alguns estudos identificaram que o alongamento agudo préexercício não altera o desempenho funcional. Desses, destacase 0 estudo de Young e Elliott ${ }^{23}$, que não encontraram diferença significativa no desempenho do salto vertical após a realização de alongamentos fundamentados nas técnicas de FNP.

$\mathrm{O}$ ponto comum entre todos os estudos destacados até 0 momento é que foi avaliado o efeito do alongamento (estático ou dinâmico) apenas sobre a musculatura agonista à atividade. Entretanto, sabe-se que os movimentos e gestuais desportivos também dependem da ação integrada dessa musculatura com os músculos antagonistas, para promover a realização da atividade de forma segura e com menor risco de lesões ${ }^{1}$. Assim, avaliar o efeito do alongamento sobre essa musculatura parece uma condição necessária para implementar programas de treinamento/tratamento mais adequados aos atletas e à população praticante de atividade esportiva.

Dentro desse contexto, Sandberg et al. ${ }^{1}$ avaliaram o efeito do alongamento agudo na musculatura antagonista (flexores de joelho, flexores de quadril e dorsiflexores do tornozelo) em 16 homens durante atividades de salto vertical e em dinamometriaisocinética. Verificaram uma melhora significativa na produção de torque na extensão de joelho em alta velocidade, assim como na potência, altura e velocidade do salto, confirmando a hipótese fundamentada em um princípio neural de inibir a ação de uma contração excêntrica da musculatura antagonista (por meio do alongamento estático) e, consequentemente, otimizar a força e potência na musculatura agonista.

Dentro desta mesma perspectiva, Ruan et al $^{19}$ investigaram a influência do alongamento estático de isquiotibiais no desempenho e no risco de lesão do ligamento cruzado anterior (LCA) durante tarefas de parada seguida de salto e corte em $180^{\circ}$. Este estudo mostrou que o alongamento agudo da musculatura antagonista favoreceu o desempenho, aumentando significativamente a altura do salto vertical devido à redução da co-contração de isquiotibiais e quadríceps durante as tarefas. Segundo os autores, a técnica de alongamento não apresentou aumento do risco de lesão do LCA, durante as tarefas realizadas. Entretanto, destaca-se que ainda são necessários novos estudos a respeito desta área abrangente e controversa, investigando o efeito do alongamento agudo na prevenção de lesões, o que não foi o objetivo do presente estudo.

Alguns fatores podem ser considerados como limitações do nosso trabalho. O primeiro deles refere-se ao reduzido tamanho amostral $(n=10)$, que impede conclusões mais amplas sobre a relação entre 0 alongamento muscular e o desempenho funcional. Entretanto, outros estudos ${ }^{9,12,18,19}$ utilizaram tamanhos amostrais similares. Ainda, o sistema de avaliação por meio de cronômetros e trenas pode estar sujeito a algum tipo de erro de medida, recomendando a utilização de instrumentos mais precisos. Contudo, no presente estudo as medidas foram realizadas sempre pelo mesmo avaliador, devidamente treinado, a fim de reduzir esse tipo de viés. Salienta-se ainda que a metodologia utilizada procurou empregar testes e instrumentos de medida de uso comum do fisioterapeuta, a fim de realizar maior aproximação a sua prática clínica/esportiva. Por fim, sugere-se realizar mais estudos abordando os efeitos do alongamento da musculatura antagonista no desempenho funcional, uma vez que as evidências encontradas na literatura ainda parecem insuficientes.

\section{Conclusão}

A realização do alongamento agudo na musculatura agonista e antagonista parece não interferir na performance dos atletas em atividades de salto. Portanto, não podemos afirmar que essa técnica leva a uma piora da performance, tal como indicado por diversos estudos.

\section{Referências}

1. Sandberg JB, Wagnerd R, Willardson JM, Smith GA. Acute effects of antagonist stretching on jump height, torque, and electromyography of agonist musculature.J StrengthCond Res 2012; 26(5):1249-56.

2. Ramos GV, Santos RR, Gonçalves A. Influência do alongamento sobre a força muscular: uma breve revisão sobre as possíveis causas. Rev Bras CineantropomDesempenho Hum 2007; 9(2):203-6.

3. Sady SP, Wartman M, Blanke D. Flexibility training: ballistic, static or proprioceptive neuromuscular facilitation?.ArchPhysMedReabil 1982 63(6):261-3

4. Hall MC, Brody, TL. Exercícios terapêuticos: na busca da função. Rio de Janeiro: Guanabara Koogan; 2001.

5. Bandy WD, Sanders, B. Exercício terapêutico, técnicas para intervenção. Rio de Janeiro: Ed. Guanabara Koogan, 2003.

6. Dacardia CF, Silva MJ. Efeitos agudos do alongamento estático no rendimento de testes funcionais em atletas de futebol.Rev Ter Man2011; 9(45):503-507.

7. O'Connor DM, Crowe MJ, Spinks WL. Effects of static stretching on leg power during cycling.J Sports MedPhys Fitness 2006; 46(1):52-56.

8. Silveira RDND, Farias JMD, Alvarez BR, Vieira J. Efeito agudo do alongamento estático em músculo agonista nos níveis de ativação e no desempenho da força de homens treinados. RevBrasMed Esporte 2011; 17(1):26-30.

9. Tricoli V, Paulo AC. Efeito agudo dos exercícios de alongamento sobre o desempenho de força máxima.Rev Bras AtivFís Saúde 2002; 7(1):6-13.

10. Shrier I. Does Stretching Improve Performance: A Systematic and Critical Review of the Literature.Clin J Sport Med 2004; 14(5):267-273. 
11. Simic L, Sarabon N, Markovic G. Does pre-exercise static stretching inhibit maximal muscular performance? A meta-analytical review. Scand J Med Sci Sports 2013; 23:131-148

12. Endlich PW, Farina GR, Gonçalves WLS, Moysés MR, Mill JG, Abreu GRD. Efeitos agudos do alongamento estático no desempenho da força dinâmica em homens jovens.Rev Bras Med Esporte 2009; 15(3):200-203.

13. Fowles JR, Sale DG, MacDougall M. Reduce strength after passive stretch of the human plantar flexores. J Appl Physol 2000; 89(5):1179-88.

14. Wilson G, Elliot B, Wood G. Stretching shorten cycle performance enhancement through flexibility training. MedSci Sports Exerc 1992; 24:116-23.

15. Barbanti VJ, Ugrinowitsch C. O ciclo de alongamento e encurtamento e a "performance" no salto vertical. Rev Paul EducFis 1998; 12:85-94.

16. Simão R, Giacomini MB, Dornelles TS, Marramon MG, Viveiros LE. Influência do aquecimento específico e da flexibilidade no teste de $1 \mathrm{RM}$. Rev Bras FisiolExerc 2003; 2:134-40.

17. Behm DG, Bambuery A, Cahill F, Power K. Effect of acute static stretching on force, balance, reaction time a movement time. MedSci Sports Exerc 2004: 36:1397-402.

18. Miranda $\mathrm{H}, \mathrm{Paz} \mathrm{GA}$, Antunes $\mathrm{H}$, Maia MDF, Novaes JDS. Efeito agudo do alongamento estático nos antagonistas sobre o teste de repetições máximas para os músculos agonistas. R Bras Ci e Mov 2014; 22(2):19-26.

19. Ruan M, Zhang Q, Wu X.Acute Effects of Static Stretching of Hamstring on Performance and Anterior Cruciate Ligament Injury Risk During StopJump and Cutting Tasks in Female Athletes. J Strength Cond Res 2017; 31(5):1241-1250.

20. Bolgla LA, Keskula DR. Reliability of lower extremity functional performance tests. J Orthop Sports Phys Ther 1997; 26(3):138-42.

21. Wordell, TW, Smith, TL, Winegardner, J. Effect of hamstring stretching on hamstringperformance. J Orthop Sports Phys Ther1994; 20(3):154-159.
22. Shrier, I. Stretching before exercise does not reduce the risk of local muscle injury: a critical review of the clinical e basic science literature. Clin J Sport Med1999; 9(4):221-227.

23. Young W, Eliott S. Acute effects of static stretching,proprioceptive neuromuscular facilitation stretching,and maximum voluntary contraction on explosive forceproduction and jupping performance.Res $Q$ Exerc Sport2001; 72(3):273-279

24. Magnusson P, Renstrom P. The European College of Sports Sciences Position statement: the role of stretching exercises in sport.Eur J Spor Sci.2006; 6(2):87-91.

25. Behm DG, Chaouachi A. A review of the acute effects of static and dynamic stretching on performance. Eur J Appl Physiol 2011; 111(11):2633-51.

26. Burkett LN, Phillips WT, Ziuraitis J. The best warm-up for the vertical jump in college-age athletic men. J Strength Cond Res2005;19(3):673-676.

27. Cramer JT, Housh TJ, Weir JP, Johnson GO, Coburn JW, Beck TW. The acute effects of static stretching on peak torque, mean power output, electromyography, and mechanomyography.Eur J Appl Physiol 2005; 93(56):530-539.

28. Unick J, Kieffer HS, Chessman W, Feeney A. The acute effects of static and ballistic stretching on vertical jump performance in trained women.J Strength Cond Res 2005; 19(1):206-212.

29. Wiktorsson-Möller M, Öberg B, Ekstrand J, Gillquist J. Effects of warming up, massage, and stretching on range of motion and muscle strength in the lower extremity. Am J Sports Med1983; 11:249-252.

30. Di Alencar TAM, Matias KFDS. Princípios fisiológicos do aquecimento alongamento muscular na atividade esportiva. RevBrasMed Esporte 2010; 16(3):230-234 